\title{
UAV PHOTOGRAMMETRY: BLOCK TRIANGULATION COMPARISONS
}

\author{
Rossana Gini $^{\mathrm{a}}$, Diana Pagliari ${ }^{\mathrm{b}}$, Daniele Passoni ${ }^{\mathrm{b}}$ Livio Pinto $^{\mathrm{b}}$, Giovanna Sona ${ }^{\mathrm{a} *}$, Paolo Dosso ${ }^{\mathrm{c}}$ \\ ${ }^{a}$ Politecnico di Milano, DICA, Geomatics Laboratory at Como Campus - Via Valleggio, 11 - 22100 Como (IT), \\ (rossana.gini, giovanna.sona)@ polimi.it \\ ${ }^{\mathrm{b}}$ Politecnico di Milano, DICA - Piazza Leonardo da Vinci 32 - 20133 Milano (IT), \\ (diana.pagliari, daniele.passoni, livio.pinto)@polimi.it \\ ${ }^{c}$ Studio di Ingegneria Terradat, via A. Costa 17 - 20037 Paderno Dugnano (IT), p.dosso@terradat.it
}

KEY WORDS: Aerial Triangulation, Software Comparison, UAV, DEM, Point cloud

\begin{abstract}
:
UAVs systems represent a flexible technology able to collect a big amount of high resolution information, both for metric and interpretation uses. In the frame of experimental tests carried out at Dept. ICA of Politecnico di Milano to validate vector-sensor systems and to assess metric accuracies of images acquired by UAVs, a block of photos taken by a fixed wing system is triangulated with several software. The test field is a rural area included in an Italian Park ("Parco Adda Nord"), useful to study flight and imagery performances on buildings, roads, cultivated and uncultivated vegetation.

The UAV SenseFly, equipped with a camera Canon Ixus 220HS, flew autonomously over the area at a height of $130 \mathrm{~m}$ yielding a block of 49 images divided in 5 strips. Sixteen pre-signalized Ground Control Points, surveyed in the area through GPS (NRTK survey), allowed the referencing of the block and accuracy analyses. Approximate values for exterior orientation parameters (positions and attitudes) were recorded by the flight control system.

The block was processed with several software: Erdas-LPS, EyeDEA (Univ. of Parma), Agisoft Photoscan, Pix4UAV, in assisted or automatic way. Results comparisons are given in terms of differences among digital surface models, differences in orientation parameters and accuracies, when available. Moreover, image and ground point coordinates obtained by the various software were independently used as initial values in a comparative adjustment made by scientific in-house software, which can apply constraints to evaluate the effectiveness of different methods of point extraction and accuracies on ground check points.
\end{abstract}

\section{INTRODUCTION}

Aerial surveys carried out by Unmanned Aerial Vehicles (UAVs) are nowadays under quick expansion, also thanks to the development of new platforms and sensors (more effective and safer) and the improvement of data acquisition devices as well as automatic systems for planning and controlling the flights.

The increased ease of use, as a consequence, widens the employment of UAVs for proximal sensing for both metric and interpretation purposes, and the capabilities of these systems are widely explored and studied according to different requirements.

As regards image sensors, the limited payload implies the use of compact digital cameras which are able to acquire a large amount of images at a very high resolution, even if often affected by higher deformations compared with those of photogrammetric calibrated cameras.

Digital images from UAVs can be processed by using the traditional photogrammetric method or software coming from the Computer Vision (CV) field: in the first case, high accuracy in points coordinates determination and in $3 \mathrm{D}$ modelling is the main pursued requirement, whilst the others work mainly to achieve a quick processing and an effective final product. In the photogrammetric approach, exterior orientation (EO) parameters and points ground coordinates are estimated together with the related accuracies: however, some difficulties often arise during the images georeferencing and the block formation phase (Aerial Triangulation), especially when images positions and attitudes are far from those commonly realized in a photogrammetric survey (aerial, terrestrial or close range).
On the other hand, by using software coming from computer vision, the processing of a large amount of images is usually faster and easier and digital model of the object, orthoimages and photorealistic 3D representations are produced with minor control on some processing steps (as georeferencing and block formation) and on the accuracies of computed geometric parameters.

Therefore, it is still necessary to test and compare the capabilities of different systems, in order to carefully assess the accuracies of final products and be aware in the choice of the system, which should be the most suitable for the survey purpose (Remondino et al. 2012).

At Dept. ICA of Politecnico di Milano, some tests are under development to validate vector-sensor systems and optimize the UAVs survey for 3D modelling. First experiments started in 2010 within the FoGLIE project (Fruition of Goods Landscape in Interactive Environment) (Gini et al., 2012), that made use of aerial imagery acquired by UAVs to enhance the natural, artistic and cultural heritage.

In this frame, images taken by compact cameras mounted on drones are processed by "traditional" photogrammetric software (PhotoModeler, Erdas LPS) and home-made software like EyeDEA realized by University of Parma (Roncella et al., 2011), Calge realized by the Dept. ICA of Politecnico di Milano (Forlani, 1986) or by software specifically built for managing UAVs images, as Pix4UAV and AgiSoft Photoscan.

This paper describes a test performed with a fixed wing system SenseFly SwingletCAM in a rural area of northern Italy and discusses the obtained results. 


\section{TEST AREA AND DATA CAPTURE}

The test flight was performed on a small test area located near Cisano Bergamasco (BG, Italy), belonging to the protected park "Parco Adda Nord" in Lombardy and already studied in the frame of FoGLIE project. The selected area of roughly $0.3 \mathrm{~km}^{2}$ comprises some buildings, secondary roads, cultivated fields and natural vegetation (Figure 1).

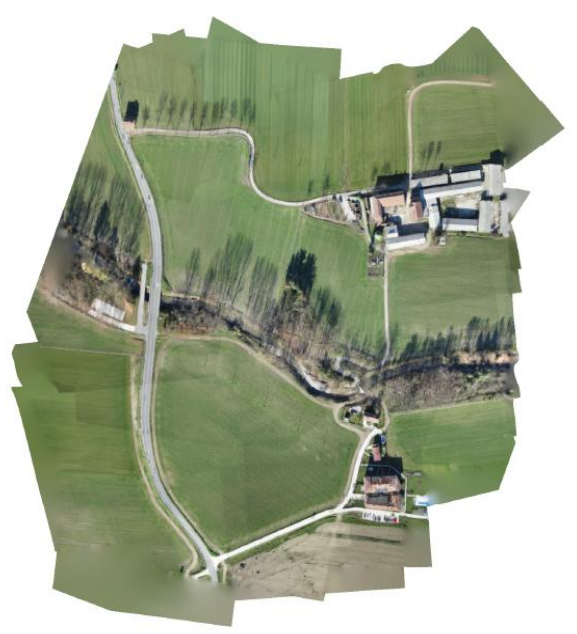

Figure 1 - Overview of the flown area

The employed UAS is a lightweight fixed wing SwingletCAM system produced by the Swiss company SenseFly (now part of the Parrot group), owned and operated by "Studio di Ingegneria Terradat".

Because of its very limited weight $(<500 \mathrm{~g})$ and size, autopilot smartness and ease of use, it is a suitable option to perform photogrammetric flights over limited areas at very high resolutions (3-7 $\mathrm{cm}$ of GSD). The SwingletCAM is able to perform pre-planned flights in a fully automated mode, though the operator can always recover full control of the system itself. Moreover, the SenseFly autopilot continuously analyzes data from the onboard GPS/IMU and takes care of all aspects of the flight mission: the SwingletCAM takes off, flies and lands fully autonomously. The system incorporates a compact camera Canon Ixus 220HS (12 Mp and fixed focal length of $4.0 \mathrm{~mm}$ ), capable of acquiring images with GSD of 3-7 cm depending on flight height.

To reach the target resolution of $4.5 \mathrm{~cm}$ GSD, the flight average altitude was set at $132 \mathrm{~m}$ AGL; furthermore, in order to gain maximum stereoscopy and avoid holes, the flight planning was performed with longitudinal and lateral overlap equal to $80 \%$. Following this approach, seven strips were necessary to cover the area of interest; however, due to strong winds and turbulences in the area during the flight, the mission was aborted several times and a subsequent rearrangement of the flight plan limited the final acquisition to 5 strips and 49 images in total. As a consequence, overlapping between strips resulted lower than the planned one, even if sufficient to guarantee a stereoscopic coverage of the whole area, as shown in Figure 2.

Big differences are present among images attitudes and positions, thus resulting in high variability in local image scale and high multiplicity of homologous points.

During the flight, the SenseFly control system recorded position and attitude of the vehicle at each shot position, thus yielding approximate values for all the Exterior Orientation parameters.

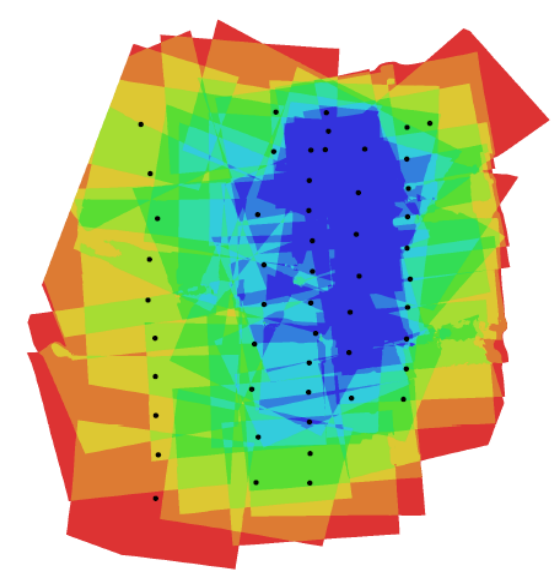

Figure 2 - Camera locations and image overlaps.

For the block georeferencing and the subsequent accuracies analysis, sixteen pre-signalized Ground Control Points (GCPs) were distributed along the edges and in the middle of the area and their centre coordinates were measured by GPS (Trimble 5700) in NRTK survey; then, a subset of these were used as Check Points (CPs).

\section{TIE POINTS EXTRACTION}

As mentioned in the introduction, the images acquired with the SenseFly vector were processed using different software. The results were analysed both in terms of EO as well as in terms of the obtained products (DSM and orthophoto).

The employed software can be divided into two main categories: "traditional" photogrammetric software and computer vision based software.

In the first group, software that follows a traditional workflow can be found: first of all, it is necessary to perform the camera calibration; then the GCPs identification and the Tie Points (TPs) research (automatic or manual, in dependence on the specific tested program) are accomplished. After that, the images are oriented (with or without self-calibration refinement) and the subsequent DSM production and the images projection for the orthophoto generation are realized.

In this context, Erdas Leica Photogrammetry Suite (LPS) and the scientific software EyeDEA were analyzed.

In the second group, 3D modelling software packages can be found : they carry out the image relative orientation together with the self-calibration, in an arbitrary reference system, which is often obtained using a minimum constraint coming from the approximate orientation provided by the telemetry. The TPs extraction, their measurement and the error rejection are completely automatized steps; the subsequent use of GCPs allows to translate and rotate the photogrammetric block in a specific reference system. Pix4UAV Desktop (from now on P4) by Pix4D 2013 and Agisoft Photoscan (from now on AP) by AgiSoft LLC 2010 were taken under analysis.

A specific procedure was realized for each software package, used independently by each other, according to its characteristic, as briefly presented below.

LPS is a photogrammetry system available in a user-friendly environment that guarantees photogrammetry results. LPS provides tools for manual and automated precision measurement and for delivering complete analytical triangulation, digital surface model generation, orthophoto production, mosaicking, and 3D feature extraction. With its 
tight integration with ERDAS Image software, LPS is a photogrammetric package for projects involving various types of data and further processing and analyses of airborne imagery. For this work the tie points used to orient the image were manually selected and measured, for a total of 295 points with an average multiplicity of 5 .

On the other hand, EyeDEA is a scientific software developed by the University of Parma and it implements SURF operator and SURF feature descriptor (Bay et al., 2008). Like any other interest operator, SURF allows to identify a large number of matches with erroneous correspondence within each set: for this reason, EyeDEA implements also some robust error rejection methods.

First of all the fundamental matrix $\mathrm{F}$ is used to define the constraint between two sets of coordinates: since the epipolar constraint is not sufficient to discriminate wrong matches between two points located on the epipolar line, also the trifocal tensor has been implemented. The RANSAC paradigm (Fischler and Bolles, 1981) is run after each geometric control to guarantee a higher percentage of inlier.

As input EyeDEA requires undistorted images, whose deformations were removed according to the parameters estimated with the camera calibration procedure implemented in the commercial software PhotoModeler V.6.33 (from now on PM). As the number of tie points extracted with EyeDEA was too large $\left(21^{\prime} 224\right)$, it was decided to reduce them to better manage the photogrammetric block during the orientation phase. The points reduction was performed with an ad hoc developed Matlab function, on the basis of the criteria of homogeneous distribution throughout the block and higher point multiplicity. In this way the final accuracy is not affected although the time required to compute the solution is significantly decreased; thus, the number of tie points was reduced to 2924 image points.

EyeDEA proceeds by successive image triplets, so the homologous points are seen, on average, only on three frames.

Since the longitudinal overlap through the block was not always adequate to guarantee the automatic extraction of points on all the subsequent triplets and in order to strengthen the block itself, the tie points extraction was also repeated along the transverse direction. Despite that, the software was not able at all to extract points on two images and for other six images it was necessary to manually measure some homologous points, because their arrangements was not good enough to ensure a bundle block adjustment solution. These operations were carried out with the commercial software PM in order to obtain the terrain coordinates necessary to the subsequent analysis and to manually measure the GCPs.

For what concerns the CV-based software, the specifically designed software for UAV application, Pix4UAV, was tested. It allows to compute the block orientation in a fully automatic way, requiring as input only camera calibration parameters and an image geo-location; moreover, GCPs were manually measured with the aim of comparing the results with the ones computed with the other programs. The coordinates of all the points used to perform the bundle block adjustment were exported and converted in the PM input format in order to generate the corresponding coordinates in the terrain reference system: these coordinates had to be used as approximate values for the next computations (see paragraph 4). The software allows to create in an automatic way also the points cloud, the DSM and the orthophoto (with a resolution of $20 \mathrm{~cm}$ ).

Eventually, AP was employed to automatically compute both image orientations and tie points cloud. In this case the software extracted a large amount of points, so it was decided to decimate them considering only the points matched at least on three images, and that yield an RMSE in AT lower than 0.40 meters. The layouts of the TPs used in the four different software are represented in Figure 3.

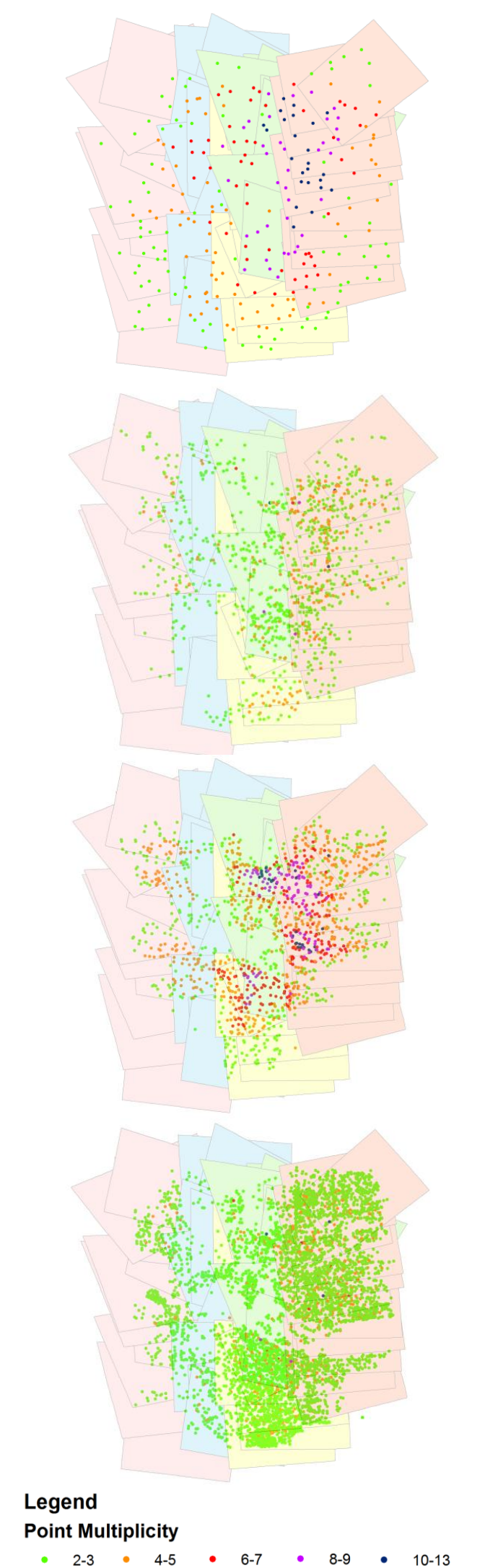

Figure 3 - Tie points distribution on the images for the different software: from top to bottom LPS, EyeDEA, P4, AP 
It is evident how the points extracted by AP outnumber the other considered cases even if they are almost all characterized by a multiplicity equal to three. The result of EyeDEA is similar in terms of multiplicity, but the set selected is smaller because the TPs were decimated before the bundle-block adjustment phase. P4 identified less points than AP but it was able to detect points visible on more images. The case of LPS is different because all the measurements were performed manually, by leading to an average multiplicity of five. A common point of all the tested software packages is that they extracted few TPs in the central zone of the block, characterized by the presence of forest trees.

\section{BUNDLE-BLOCK ADJUSTMENT}

Considering the different nature of the software, it was decided to uniform the EO analysis by defining a standard procedure: for this purpose the scientific software Calge was used. Calge is an home-made computer program designed to realize bundle block compensation of a general topographic network or of a photogrammetric block.

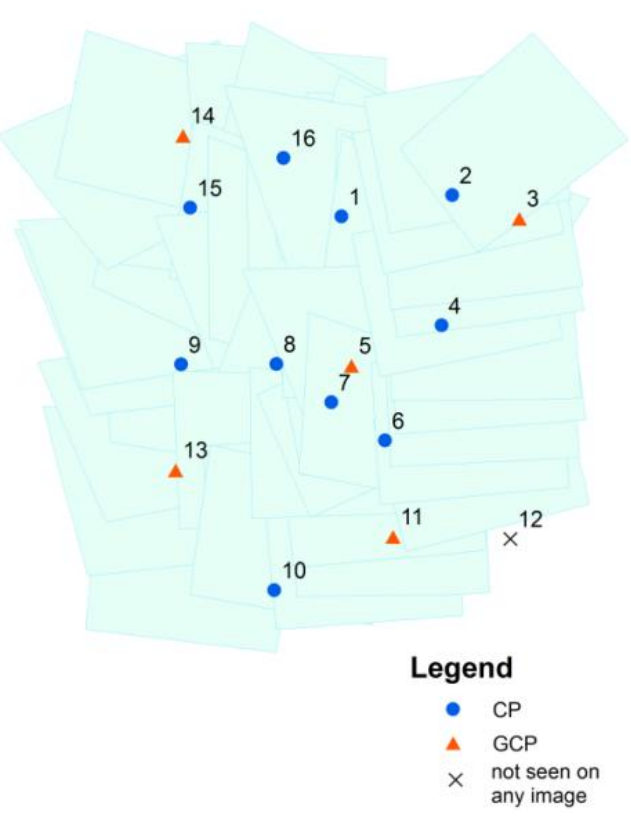

Figure 4 - The 10 CPs employed in the analysis with 5 GCPs

A first comparison between the different software involved the bundle-block adjustment using the TPs measured, either manually (LPS, some points for EyeDEA and all the GCPs) or automatically (the most of TPs extracted with EyeDEA and all the points identified by $\mathrm{P} 4$ and AP). In all cases, the calibration parameters were refined thank to the self-calibration executed during the bundle-block adjustment itself: especially, the variations of the 10 parameters of the Fraser model (Fraser, 1997) were estimated. For each software, two different kinds of bundle-block adjustment were realized: i) constraining all the measured GCPs; ii) constraining only 5 GCPs, 4 of which selected along the block edges and one near the center (see Figure 4). Both GCPs and CPs measures on images were done manually by different non-expert operators.

In Table 1 the obtained results are listed.

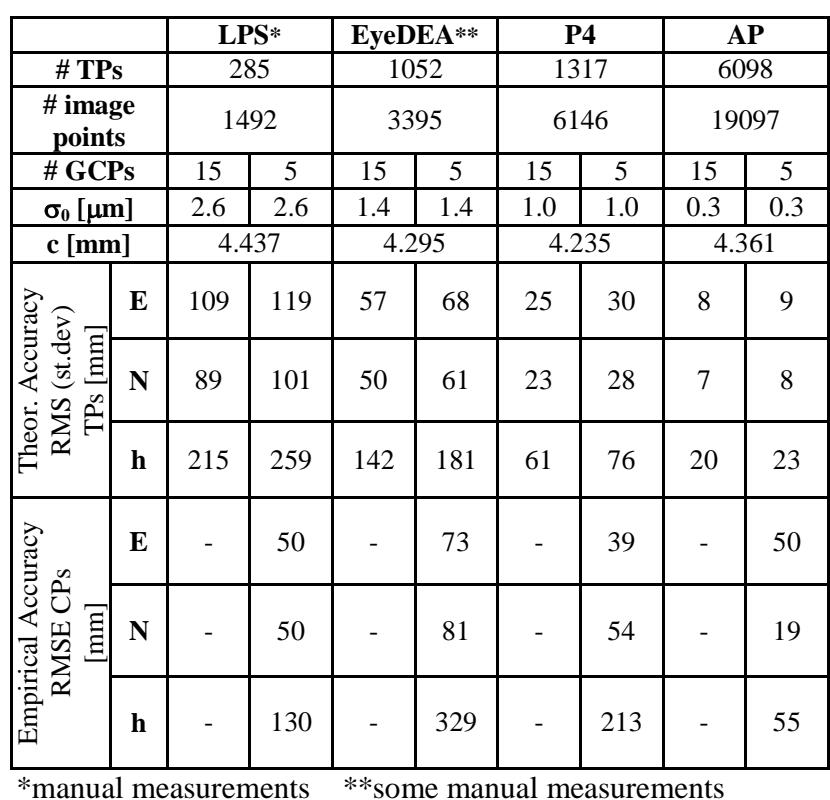

Table 1 - Bundle-block adjustment results (15 and 5 GCPs configuration)

The first rows show the number of TPs and the observation sample sizes: the ratio between these two quantities is equal to the average TPs multiplicity.

In the subsequent row, the bundle-block $\sigma_{0}$ is reported: it ranges from $0.3 \mu \mathrm{m}$ (AP) to $2.6 \mu \mathrm{m}$ (LPS), respectively 0.2 and 1.6 times the pixel size equal to $1.54 \mu \mathrm{m}$.

The estimated focal lengths, in the fifth row, vary between $4.235 \mathrm{~mm}$ (P4) and $4.437 \mathrm{~mm}$ (LPS), representing meaningful corrections with respect to the initial calibrated value of 4.3559 $\mathrm{mm}$. On the other hand, the self calibrated values do not vary significantly, also respect to estimated accuracies that are of the same order of magnitude. Moreover, as images were taken at constant height there is a large correlation between estimated focal length $\mathrm{c}$ and estimated projection centers heights $\left(\mathrm{Z}_{0}\right)$ : a variation in $\mathrm{c}$ is absorbed by variation in $\mathrm{Z}_{0}$.

In the following rows the RMS of standard deviation of the TPS and RMSE of the CPs are shown. As expected, the RMS of the standard deviation values are smaller for software that extracted TPs automatically (also due to the lower value of $\sigma_{0}$ ). As regards CPs RMSE, results are more homogenous, especially in East and North coordinates that are around GSD; the differences are more pronounced in altitude.

A further analysis was carried out by using Calge to evaluate the quality of the EO parameters for each software. Since EyeDEA performed only the TPs extraction, the EO parameters were calculated by PM. The analyses were realized in a consistent way because it was decided to constrain the EO parameters obtained using only 5 GCPs. At the same time also a self-calibration was performed, in order to evaluate the best calibration parameters set. The RMSE of CPs residuals are summarized in Table 2.

\begin{tabular}{|c|c|c|c|c|}
\hline & LPS & $\begin{array}{c}\text { EyeDEA } \\
\text { /PM }\end{array}$ & P4 & AP \\
\hline East $[\mathrm{mm}]$ & 48 & 16 & 81 & 74 \\
\hline North $[\mathrm{mm}]$ & 47 & 12 & 46 & 61 \\
\hline height $[\mathrm{mm}]$ & 90 & 36 & 214 & 83 \\
\hline
\end{tabular}

Table 2 - RMSE on the CPs residuals obtained in the second test (fixed EO) 
The RMSE values are low with respect to the image scale of 1:31'000 and the GSD equal to $4.5 \mathrm{~cm}$. Considering the horizontal coordinates, the minimum value $(0.33 * \mathrm{GSD})$ was achieved with the combination of the software PM and EyeDEA, followed by LPS ( 1 *GSD). Worse results were obtained by $\mathrm{P} 4$ and AP.

Considering the height coordinates, the RMSE are higher than horizontal ones, even if the values are smaller than $100 \mathrm{~mm}$ (with the exception of the value obtained by processing the block with $\mathrm{P} 4$, which is equal to $214 \mathrm{~mm}$ ).

\section{DSM COMPARISONS}

A second kind of comparison among software results was done analyzing the DSM they produced. A mesh of $0.20 \mathrm{~m}$ was chosen to compute surface models with different software; automatic procedures were used in LPS, P4 and AP whilst another home-made software called Dense Matcher (Re et al., 2012) was used to process the data coming from EyeDEA/PM workflow. The points cloud created by DM was interpolated on the same grid mesh through ArcGIS 10.0. A first visual analysis shows a different behavior where sharp height variations occur, for instance, around buildings. P4, DM and LPS indeed compute interpolated values, as in all the other parts, while AP, run in "sharp" mode, seems to recognize edges and produces a sharper DSM (see Figure 5).
AP

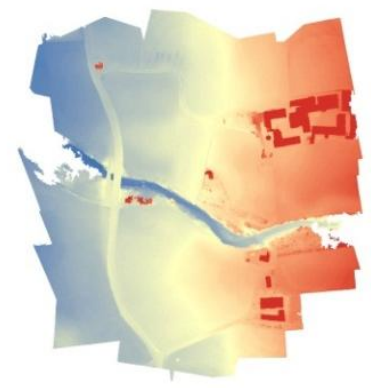

LPS

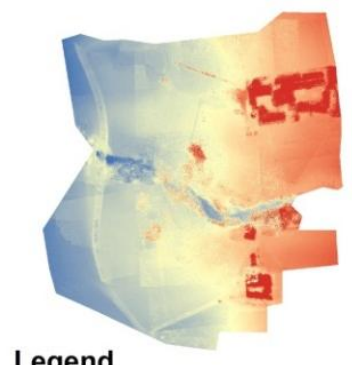

Legend

height ( $\mathrm{m})$

High : 270
Low : 231
P4

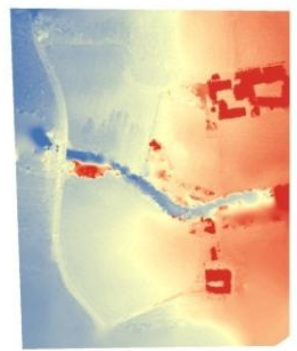

DM

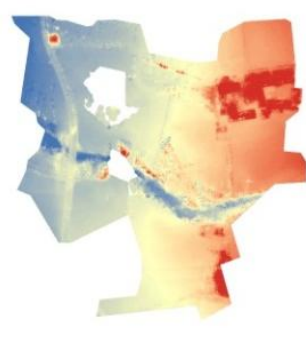

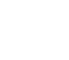

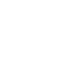

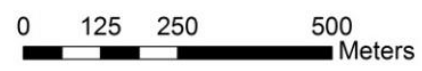

Figure 5 - DSM from AP, LPS, P4 and DM

This is clearly visible in the layouts (see Figure 6) where the differences coming from AP and the other software are presented. Statistics of the differences yield an average value of some centimeters and a standard deviation of 84,89 and $103 \mathrm{~cm}$ respectively for P4-AP, DM-AP and LPS-AP differences. The maximum absolute values are about $20 \mathrm{~m}$ near building edges and in the central area covered by very high trees.
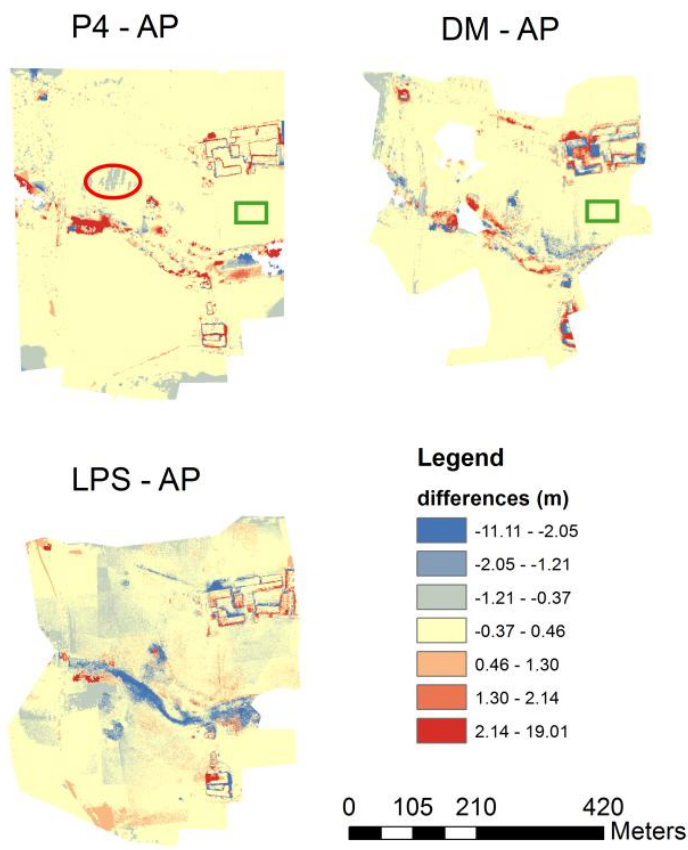

Figure 6 - DSMs differences

In most areas (about 90\%) differences are in the range of -0.3 $\mathrm{m}, 0.4 \mathrm{~m}$. The average of the differences close to zero shows the absence of vertical and horizontal biases for all DSMs.

A detailed analysis made on a regular and flat area (green line in Fig.6) confirmed the difference in smoothing effect between the surface generating approaches (see Figure 7). In this case, the maximum variations are about $50 \mathrm{~cm}$ with an average of $4.2 \mathrm{~cm}$.

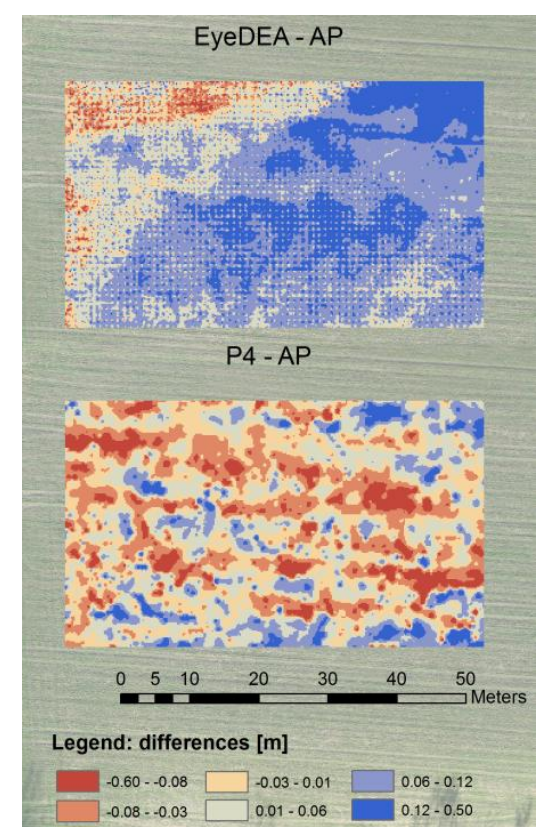

Figure 7 - Differences of DSMs: detail in a flat area

In P4-AP comparison, the anomalous behavior visible in the red circle is due to the presence of trees' shadows (see Figure 8): the almost flat ground was modeled in one case with false height variations of the order of $1 \mathrm{~m}$. This is probably due to homologous points chosen at shadows edges, which are slightly moving during the survey, thus causing mismatching and false intersections. This effect is visible also in the LPS and DM 
DSMs. Again, there's a different software behavior: P4 and the other software produced higher and sharper undulations, while AP gave a smoother surface.

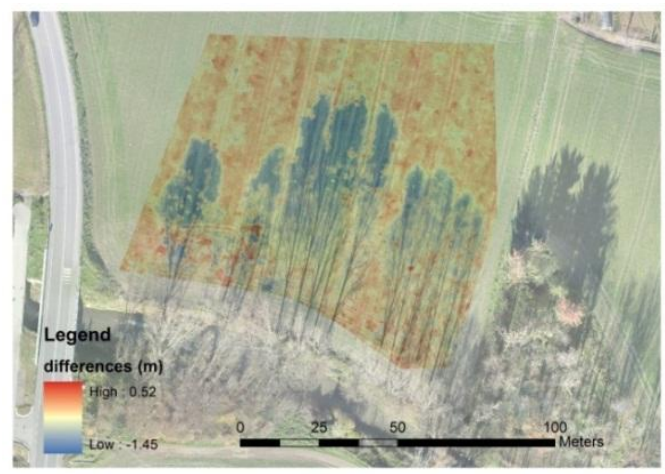

Figure 8 - Differences of DSMs (P4-AP): detail in shadow area produced from trees

Finally, Figure 9 shows the two ortophotos carried out from the DSM generated by P4 (up) and AP (down). The different behavior near the roof edges is clear: AP defined the edges better than $\mathrm{P} 4$.

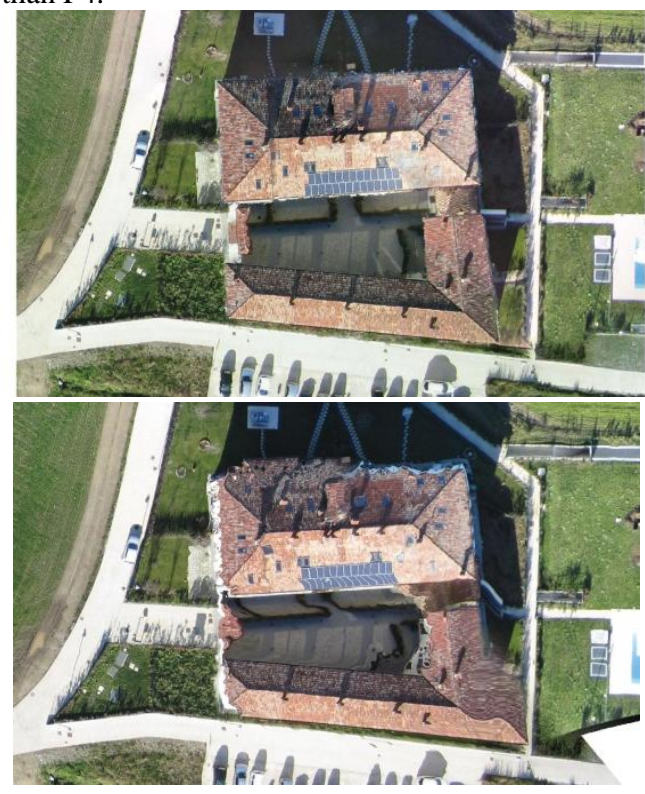

Figure 9 - Differences of ortophotos (AP up - P4 down): detail in the edges of some buildings

\section{CONCLUSIONS}

The images acquired by UAVs, in particular the fixed-wing ones, are suitable to be processed by different software packages: in particular, both computer vision-based and photogrammetric software (even home-made like EyeDEA and DM) was analyzed in this paper. The whole set was able to provide the images exterior orientation and products such as the DSM, although programs of the first type can work almost entirely in an automatic way as well as they can quickly create a high quality final product; moreover, both P4 and AP can automatically generate very dense point clouds with high multiplicity. The photogrammetric software requires an operator's intervention in some phases, as in the Exterior Orientation validation, in the estimation of the self-calibration parameters or in the manual selection of points in critical areas of the images. The computational time is often very high in comparison with the other software: for instance, the DSM generation in DM required many hours of processing. On the other hand, the photogrammetric software results are better (see Table 2), in terms of CPs RMSE obtained by constraining the EO parameters. Thanks to the DSMs analyses, it can be said that the implemented strategy of AP seems to be able to achieve the most reliable results: this is highlighted by a details comparison rather than a global analysis (indeed, all products did not have systematic errors); moreover, AP provided the best product, especially in flat areas and in the presence of shadows. Eventually, the strategy that AP employs for the buildings outlines allows the creation of orthophotos with a high level of quality (see Figure 9).

\section{REFERENCES}

\section{References from Journals:}

Bay H., Ess A., Tuylelaars T., Van Gool., 2008. Speeded Robust Features (SURF). Computer Vision and Image Understanding (110), pp. 346-359.

Fischler, M., Bolles, R., 1981. Random sample consensus: a paradigm for model fitting with application to image analysis and automated cartography. Com. Ass. Comp. Mach., Vol 24, pp. 81-95.

Re, C., Roncella, R., Forlani, G., Cremonese, G., and Naletto, G., 2012. Evaluation of area-based image matching applied to DTM generation with Hirise images, ISPRS Ann. Photogramm. Remote Sens. Spatial Inf. Sci., I-4, 209-214.

Forlani, G., 1986. Sperimentazione del nuovo programma CALGE dell'ITM. Bollettino SIFET No. 2, pp. 63-72.

Fraser, C.S., 1997. Digital camera self-calibration. ISPRS Journ. of Photogram. and Rem. Sensing, Vol. 52, pp. 149-159.

\section{References from Other Literature:}

Remondino, F., Del Pizzo, S., Kersten, T., Troisi, S., 2012: Low-cost and open-source solutions for automated image orientation - A critical overview. Proc. EuroMed 2012 Conference, M. Ioannides et al. (Eds.), LNCS 7616, pp. 40-54.

Roncella, R., Re, C., Forlani, G., 2011. Performance evaluation of a structure and motion strategy in architecture and cultural heritage, Int. Arch. Photogramm. Remote Sens. Spatial Inf. Sci., Volume XXXVIII-5/W16, pp. 285-292.

Gini, R., Passoni, D., Pinto, L., Sona, G., 2012. Aerial images from an UAV system: $3 \mathrm{~d}$ modeling and tree species classification in a park area. Int. Arch. Photogramm. Remote Sens. Spatial Inf. Sci., Vol. XXXIX-B1, pp. 361-366.

\section{References from websites:}

AgiSoftLLC.(2010).AgiSoftPhotoScan.http://www.agisoft.ru/pr oducts/photoscan/.

Pix4UAV Desktop by Pix4D, 2013.http://pix4d.com/pix4uav.ht $\mathrm{ml}$

\section{Acknowledgements}

The authors thank Riccardo Roncella for allowing them the use of the software EyeDEA and for his help and Pix4D, platinum sponsor of UAV-g 2013. 